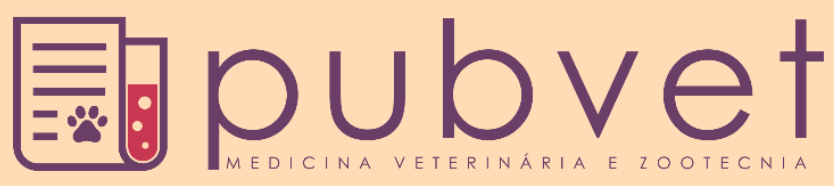

https://doi.org/10.31533/pubvet.v13n4a304.1-11

\title{
Efeitos da torta de neem no controle alternativo de nematoides gastrintestinais em ovinos: Revisão
}

\author{
Renan da Silva Fonseca ${ }^{\bullet}$, Aparecida de Fatima Madella de Oliveira $^{2}{ }^{\bullet}$, Isabella Vilhena Freire \\ Martins $^{3 \oplus}$, Dirlei Molinari Donatele ${ }^{3}$, Fabricio Albani de Oliveira $^{4}{ }^{\oplus}$, Maria Larissa

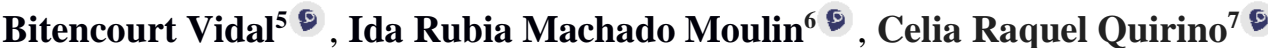 \\ ${ }^{1}$ Mestrando do curso de Mestrado Profissional em Agroecologia do Instituto Federal do Espírito Santo Campus de Alegre, Brasil \\ ${ }^{2}$ Professora do Instituto Federal do Espírito Santo, Alegre, ES, Brasil. \\ ${ }^{3}$ Professores da Universidade Federal do Espírito Santo, ES, Brasil \\ ${ }^{4}$ Médico Veterinário do Instituto Federal do Espírito Santo, Alegre, ES, Brasil. \\ ${ }^{5}$ Mestranda do curso de Mestrado em Ciências Veterinárias na Universidade Federal do Espírito Santo, ES, Brasil \\ ${ }^{6}$ Discente no curso de Licenciatura em Ciências Biológicas no Instituto Federal do Espírito Santo, Alegre, ES, Brasil. \\ ${ }^{7}$ Professora da Universidade Estadual do Norte Fluminense, Campos dos Goytacazes, RJ, Brasil. \\ *Autor para correspondência, E-mail: renanfonsecamedvet@gmail.com
}

Resumo. Um dos principais problemas da ovinocultura é o parasitismo causado por nematóides gastrintestinais que acarretam grandes prejuízos na atividade, e que inviabilizam a produção econômica. Por esse motivo a utilização sem critério técnico de produtos químicos se tornam cada vez mais frequentes nas propriedades, contribuindo assim para a resistência parasitária. Devido a essa resistência criada pelos parasitas aos anti-helmínticos sintéticos, é notória a necessidade de buscar novos princípios ativos eficazes no controle de endoparasitas, sendo a fitoterapia uma alternativa sustentável. Neste contexto a utilização de extratos de plantas popularmente conhecidas como neem (Azadiractha indica) que contem atividade inseticida, pode se tornar uma alternativa viável no combate aos nematoides gastrintestinais. Portanto, a proposta deste artigo é fazer uma revisão de literatura abordando o possível potencial da utilização de torta de neem (Azadiractha indica) como fonte alternativa no controle de endoparasitas na ovinocultura, minimizando a utilização de produtos químicos sintéticos, nocivos ao meio ambiente.

Palavras-chave: fitoterapia, ovinocultura, resistência parasitária

\section{Effects of neem pie on the alternative control of gastrointestinal nematodes in sheep: Review}

\begin{abstract}
One of the main problems of sheep farming is the parasitism caused by gastrointestinal nematodes that causes great losses in the activity and that makes economic production unfeasible. For this reason, the use without technical criteria of chemical products becomes more frequent in the properties, thus contributing to the parasitic resistance. Due to this resistance created by parasites to synthetic anthelmintics, the need to seek new active principles effective in the control of endoparasites is well known, with phytotherapy being a sustainable alternative. In this context the use of plant extracts popularly known as neem (Azadiractha indica) containing insecticidal activity may become a viable alternative in the fight against gastrointestinal nematodes. Therefore, the purpose of this paper is to review the literature on the possible potential of the use of neem pie (Azadiractha indica) as an alternative source for the control of endoparasites in sheep farming, minimizing the use of synthetic chemicals that are harmful to the environment.
\end{abstract}

Keywords: parasitary resistance; phytotherapy; sheep 


\section{Efectos de la torta de neem en el control alternativo de nematodos gastrointestinales en ovinos: Revisión}

Resumen. Uno de los principales problemas de la ovinocultura es el parasitismo causado
por los nematodos gastrointestinales que acarrean grandes perjuicios en la actividad, y que
inviabilizan la producción económica. Por eso la utilización sin criterio técnico de
productos químicos se vuelve cada vez más frecuente en las propiedades, contribuyendo
así a la resistencia parasitaria. Debido a esta resistencia creada por los parásitos a los
antihelmínticos sintéticos, es notoria la necesidad de buscar nuevos principios activos
eficaces en el control de endoparásitos, siendo la fitoterapia una alternativa sostenible. En
este contexto la utilización de extractos de plantas popularmente conocidas como neem
(Azadiractha indica) que contiene actividad insecticida, puede convertirse en una
alternativa viable en el combate a los nematodos gastrointestinales. Por lo tanto, la
propuesta de este artículo es hacer una revisión de literatura abordando el posible potencial
de la utilización de torta de neem (Azadiractha indica) como fuente alternativa en el control
de endoparásitos en la ganadería ovina, minimizando la utilización de productos químicos
sintéticos, nocivos para el medio ambiente.

Palabras clave: fitoterapia, ganadería, resistencia parasitaria

\section{Introdução}

O principal problema sanitário na criação de pequenos ruminantes no Brasil é causado por nematoides gastrintestinais. Os animais ao terem o organismo invadido por esses parasitos sofrem redução no ganho de peso, diminuição na taxa de fertilidade, gastos com medicamentos e aumento na mortalidade, causando significativas perdas econômicas (Nova et al., 2014; Sczesny-Moraes et al., 2010).

O controle de nematoides gastrintestinais por meio de método químico é amplamente utilizado, porém, como consequência do uso indiscriminado sem critérios técnicos vem ocorrendo o desenvolvimento de organismos cada vez mais resistentes, resultando na utilização de dosagens cada vez maiores, sem obter resultado satisfatório, além de se tornarem altamente tóxicos, sendo prejudiciais ao ambiente e à saúde humana e ao próprio animal (Amarante et al., 2004). Vieira (2008) cita que os resíduos de compostos químicos eliminados com as excreções dos animais têm sérios efeitos no meio ambiente. Chagas (2004) diz que o uso maciço de vermífugos sintéticos causa liberação de resíduos nos produtos de origem animal como carne, vísceras e leite, que são prejudiciais à saúde humana. Devido à resistência criada pelos parasitos aos anti-helmínticos sintéticos, é notória a necessidade de buscar novos princípios ativos eficazes no controle de endoparasitas, sendo a fitoterapia uma alternativa sustentável (Amarante, 2009). Segundo Chagas et al. (2008) os medicamentos naturais não deixam resíduos nocivos, apresentam menor custo de produção e são rapidamente degradáveis. Entre eles destaca-se o neem (Azadirachta indica, A. Juss), planta estudada a partir de seus componentes biológicos (Nova et al., 2014; Sczesny-Moraes et al., 2010). O neem é uma planta originária da Índia, pertencente à família das Meliaceae (Dias et al., 2014). Apresentam vários compostos biocidas, encontrados em toda a planta, elementos conhecidos como terpenóides ou limonóides, no entanto, o mais potente entre eles é a Azadiractina que possui concentrações diferentes de acordo com a parte da planta utilizada (Mossini \& Kemmelmeier, 2005). Os produtos à base de neem apresentam eficácia contra elevada quantidade de organismos maléficos seja na agricultura, na pecuária ou outras atividades. Seus princípios ativos são eficazes no controle de insetos, nematoides, caramujos, crustáceos, bactérias, vírus e fungos (Mossini \& Kemmelmeier, 2005).

O extrato de neem possui ação sobre diferentes locais no corpo dos parasitos, entre eles incluem: diminuição da ingestão de nutrientes, diminuição da postura de ovos de ectoparasitos, regulação do crescimento, inibição da reprodução, impedimento de movimentação e inibição da síntese de quitina. Essa ação acontece na maioria dos casos nas fases larvais, mas algumas espécies respondem nas suas fases adultas (Chagas et al., 2008; Mossini \& Kemmelmeier, 2005; Schmutterer, 1990). O neem pode ser utilizado de diferentes formas, entre elas destacando-se os produtos obtidos pela moagem das folhas, extratos aquosos das folhas, da extração de óleo das sementes e pela torta nem (Kudke et al., 1999). 
Contudo, dependendo da forma utilizada a concentração de Azadiractina pode variar. No processo de obtenção da torta de neem, pela prensagem das sementes, segundo Brechelt et al. (1995), 90\% da Azadiractina presente fica concentrado na torta, caracterizando um produto com maior potencial de ação.

Dias et al. (2014) em um estudo conduzido com equinos, concluíram que a utilização de $20 \mathrm{~g}$ diárias de uma formulação comercial contendo torta de neem em um período de seis meses apontou eficácia comparando com o uso somente de antihelmintico de forma periódica. Não têm sido observados muitos relatos quanto à aplicação de torta neem no controle de nematoides gastrintestinais em pequenos ruminantes. Portanto, a utilização de torta de neem no controle fitoterápico de endoparasitas de ovinos e caprinos pode ser uma alternativa sustentável para diminuir os prejuízos econômicos, além de reduzir impactos ambientais, que também refletem aos seres humanos, causados pelo uso indiscriminado de vermífugos sintéticos.

Objetiva-se nesta revisão abordar a importância do controle de parasitas em ovinos e o potencial antiparasitário do neem (Azadiractha indica) como fonte alternativa no controle integrado de nematoides gastrintestinais de ovinos.

\section{Ovinocultura no Brasil}

A ovinocultura no Brasil vem ganhando forças nos últimos anos, e segundo Aquino et al. (2016) o crescimento da ovinocultura se deve a alguns fatores importantes como manejo, melhoramento genético entre outros, entretanto o consumo de carne ovina ainda é baixo (ANUALPEC, 2018).

Outro fator que influenciou foi à descoberta dos pecuaristas que o custo de produção da carne ovina é menor que a de bovinos, e em algumas situações se torna uma atividade bem mais rentável. Aquino et al. (2016) ainda ressaltam que o custo de manutenção de dez ovelhas em uma mesma área onde se cria uma vaca é muito menor. Adicionalmente, um bovino necessita de um hectare de capim para se alimentar durante um ano e atingir entre 200 a $250 \mathrm{~kg}$. Nesta mesma área, em contrapartida, 60 ovinos podem pastejar e produzir até $900 \mathrm{~kg}$ de carne.

Segundo Viana et al. (2015), o Brasil ainda não é autossuficiente na produção de carne ovina para atender a demanda interna, levando-o assim, a importar o produto de outros países, sendo o Uruguai o principal parceiro comercial do brasil. Segundo pesquisadores da Embrapa Caprinos e Ovinos, tanto a produção quanto o consumo de produtos desses pequenos ruminantes irão aumentar no Brasil ao longo do prazo. Isso ocorrerá devido ao crescimento populacional, resultando na organização desse setor com intuito de expandir mercado, expressando seu maior potencial (Aquino et al., 2016). No entanto, existem alguns gargalos que precisam ser superados, como a questão da formalização do abate e a melhor inspeção sanitária dos produtos. Sorio \& Rasi (2010) relatam que mais de 95\% dos abates de ovinos são realizados de forma clandestina no Brasil, o que dificulta a certificação de qualidade e desmotiva o produtor a investir na profissionalização do rebanho.

Outra visão para um mercado promissor está relacionada com a qualidade, pois o consumidor não deseja carne de animais de descarte, mas sim de cordeiros abatidos precocemente. A organização social dos produtores com intuito de obterem força no mercado, atendendo a evolução e exigências dos consumidores, os ovinocultores poderão se beneficiar das vantagens do atual cenário da ovinocultura (Andrade, 2015).

O rebanho ovino no Brasil cresceu discretamente entre 2014 a 2017, finalizando o período com um rebanho efetivo de um pouco mais de 18 milhões de animais (ANUALPEC, 2018). No Sudeste houve um crescimento na população ovina de 55,6\% de 2000 a 2017, com um ápice no tamanho do rebanho em 2010, atingindo um pouco mais de 780 mil cabeças de ovinos, mas em contrapartida de 2010 à 2017 houve um pequeno declínio de 20,32\%. O consumo brasileiro de carne ovina está entre $0,6-0,7 \mathrm{~kg}$ per capita ano (ANUALPEC, 2018), um consumo considerado baixo ao comparar-se com o consumo de outras carnes como, por exemplo, bovina, suína e de frango, que chegam a ter um consumo per capita ano de $35 \mathrm{~kg}, 14,8 \mathrm{~kg}$ e 44,1 $\mathrm{kg}$ respectivamente (ANUALPEC, 2018).

A ovinocultura brasileira encontra-se em expansão, porém ainda tem muito a evoluir. $\mathrm{O}$ aumento do consumo de carne ovina é o principal desafio a ser seguido a fim de acelerar o crescimento da 
ovinocultura. Intervenções que visem aumentar o consumo devem estar atentas a estratégias de marketing que apresentem a carne ovina como sendo um produto seguro e de qualidade, além de ações que possibilitem as indústrias disponibilizarem uma ampla variedade de cortes para que todas as classes sociais possam ter acesso à carne ovina, com o intuito de, em longo prazo, fidelizar o consumidor (Viana, 2008).

\section{Nematoides Gastrintestinais em Ovinos}

Os ovinos possuem vários parasitas Gastrintestinais, entre eles; Haemonchus contortus e Trichostrongylus axei que se localizam no abomaso; Trichostrongylus colubriformis, Strongyloides papillosus, Cooperia punctata, Cooperia pectinata e Bunostomum trigonocephalum que parasitam o intestino delgado e Oesophagostomum colubianum, Trichuris ovis, Trichuris globulosa e Skrjabinema sp. que se localizam no intestino grosso (Vieira, 2008). Os endoparasitos que apresentam maior prevalência e maior intensidade de infecção, sendo considerados os nematoides de maior importância econômica para a exploração de pequenos ruminantes são os Haemonchus contortus, Trichostrongylus colubriformis, Strongyloides papillosus e Oesophagostomum colubianum, sendo o Haemonchus contortus que acarreta maior prejuízo econômico na ovinocultura mundial (Afonso et al., 2013; Vieira, 2008). Haemonchus contortus é um nematoide de grande importância na criação de ovinos, pelo fato de apresentar um alto potencial biótico e elevada intensidade de infecção. Além disso, é denominado um helminto hematófago, vive no abomaso, responsável por acarretar um quadro clínico severo de anemia e elevando os casos de mortalidade (Amarante, 2009; Schmutterer, 1990; Urquhart, 1996). Os principais efeitos patogênicos de Haemonchus contortus são causados pelos L4 (quarto estágio larval) e adultos, que se alimentam de sangue, levando a um quadro de anemia grave, que geralmente se torna aparente após duas semanas de infecção. A doença em sua forma aguda está associada a sinais de anemia hemorrágica, fezes de cor escura, edema submandibular, fraqueza, produção reduzida de lã e massa muscular, ou às vezes morte súbita, dependendo da intensidade da infecção. Em casos de doença crônica, os sinais clínicos mais observados são: diminuição da ingestão de alimentos, perda de peso e anemia (Roeber et al., 2000; Roeber et al., 2013). As larvas de primeiro, segundo e terceiro estágio (L1, L2 e L3, respectivamente) são de vida livre no meio ambiente. O quarto estágio larval (L4) e adultos são parasitos no trato gastrintestinal do hospedeiro conforme mostra a Figura 1 (Roeber et al., 2000; Roeber et al., 2013). Em um rebanho, quase todas as ovelhas estão infectadas com um ou mais desses nematoides, mas a intensidade da infecção e os sinais clínicos associados à doença podem variar consideravelmente (Sotomaior et al., 2007).

As interações entre o hospedeiro e o parasito determinam o potencial da doença e o padrão da infecção, enquanto que a interação entre ambiente-hospedeiro e parasito influenciam a transmissão da doença (Levine, 1973; Roeber et al., 2000). As regiões com variações climáticas têm grande influência sobre a epidemiologia das infecções de nematoides e sua distribuição geográfica (Roeber et al., 2000).

A distribuição de espécies e a disponibilidade sazonal de diferentes parasitos são em grande parte determinadas pelo desenvolvimento bem-sucedido de seus estágios de vida livre, com condições ambientais favoráveis, particularmente a temperatura e a umidade relativa, são de grande importância (Roeber et al., 2013). Outro fator que influencia nas infestações de parasitos gastrintestinais em ovinos, é o tipo de manejo que os animais são submetidos. O manejo semi-extensivo, o qual o animal vai a pasto, e geralmente o mesmo está rente ao solo, favorecendo as parasitoses, além da aglomeração dos animais e a baixa frequência de limpeza dos galpões onde os animais ficam alojados, contribuindo assim, a sobrevivência e o desenvolvimento das larvas favorecendo a reinfecção dos animais (Jimenez-Sanz et al., 2016). Jimenez-Sanz et al. (2016) avaliaram ovelhas em diferentes estados fisiológicos e obtiveram como resultado após o exame de coprocultura, um percentual médio de gêneros e larvas infectantes (L3) de nematoides gastrintestinais de $62 \%$ de Haemonchus spp, 36\% de Trichostrongylus spp, $1 \%$ de Cooperia spp e 1\% de Oesophagostomum spp. Jimenez-Sanz et al. (2016) afirmam que fêmeas ovinas em final de gestação e em lactação estão mais propensas a infecções por nematoides gastrintestinais, Jimenez-Sanz et al. (2016) ainda acrescentam que o método Famacha $\odot$ é uma forma prática de avaliar o grau de anemia em ovinos e indiretamente o grau de infestação por Haemonchus contortus. 


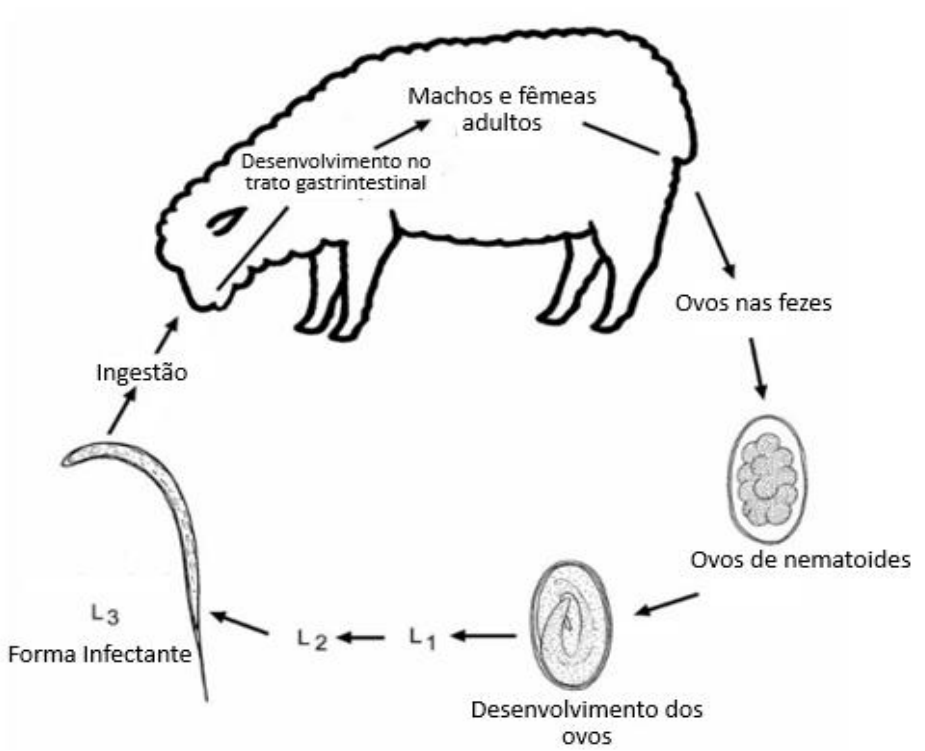

Figura 1 - Representação do ciclo de vida dos nematoides gastrintestinais (ordem Strongylida) de pequenos ruminantes. Fonte: Adaptado de Roeber et al. (2013).

Segundo Amarilho-Silveira et al. (2015) as infestações por nematoides gastrintestinais estão relacionadas a idade, estado nutricional, e as transições fisiológicas, ressaltaram ainda que em fases que exigem mais nutrientes, voltado principalmente para a produção, há uma incidência maior. Já Amarante (2009) acrescenta que as raças europeias introduzidas no Brasil apresentam grande susceptibilidade às infecções causadas por parasitas que ocorrem nos trópicos, como é o caso de $H$. contortus. Roeber et al. (2013) citam três grupos de animais que são propensos a cargas pesadas de vermes; (I) animais jovens, não imunes, (II) animais adultos, imunocomprometidos e (III) aqueles expostos a uma alta pressão de infecção do ambiente contaminado com L3. Os nematoides gastrintestinais de ovinos são transmitidos de forma horizontal e direta. A relação do parasita, hospedeiro e o ambiente é exibida na Figura 2. Muitos fatores ligados a essa relação determinam o tipo de intensidade da infecção, os fatores relacionados ao hospedeiro são idade, imunidade, sexo, espécies e resistência genética, os fatores relacionados ao parasita incluem história de vida, duração da fase histotrófica, sobrevivência das larvas no meio ambiente e sua localização no hospedeiro, e os fatores ambientais incluem clima, estação do ano, tipo de vegetação nas pastagens e microclima (Roeber et al., 2013).

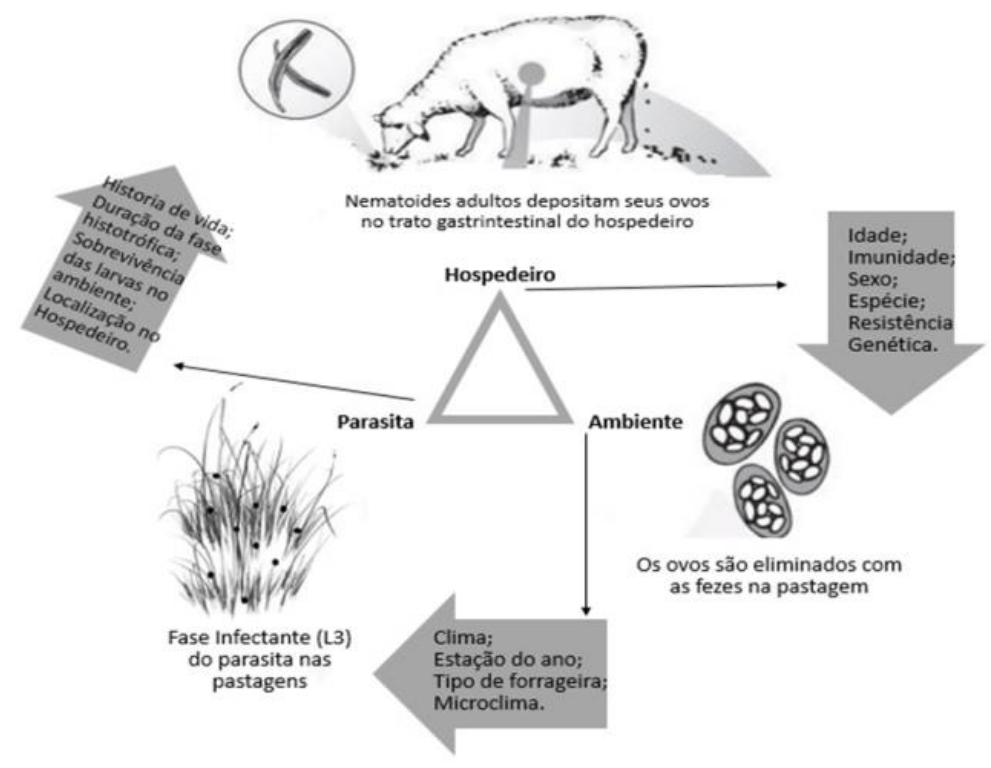

Figura 2. Relação entre hospedeiro, parasitos e meio ambiente. Fonte: Roeber et al. (2013). 
Com o aumento das populações de endoparasitas nos ovinos, os produtores adotam como medidas de controle a utilização de drogas sintéticas (anti-helmínticos). Entre tanto, os efeitos adversos do uso indiscriminado dessas drogas têm provocado o aparecimento de biótipos resistentes, fazendo com que o pecuarista utilize dosagens cada vez maiores sem obter resultados satisfatórios (Amarante et al., 2004).

\section{Resistência anti-helmíntica}

Um dos fatores primários que interferem na produção de ovinos é a alta prevalência de infecções parasitárias e a dificuldade de controle eficaz dos nematoides gastrintestinais em pequenos ruminantes, e esse fator pode comprometer o desempenho do animal, bem-estar e aumentar índices de mortalidade (Fortes \& Molento, 2013). Em rebanhos onde já foram diagnosticados problemas de resistência antihelmíntica o prejuízo econômico é maior, pois além da diminuição na produtividade do rebanho também existe os gastos na aquisição de vermífugos cuja eficácia é afetada em função da resistência parasitária (Vieira, 2008). O aparecimento de resistência seguiu a sequência em que os anti-helmínticos de amplo espectro foram lançados no mercado para uso comercial. Segundo Barbieri et al. (2014) o uso inadequado desses produtos químicos para o controle de helmintos, entre eles o Haemonchus contortus, resultou no surgimento de cepas resistentes.

A alta densidade de animais por área, o curto intervalo entre tratamentos, tratamentos supressivos, rápida alternância de diferentes princípios ativos, introdução de novos animais ao rebanho em que possuem parasitas com resistência, uso em excesso e indiscriminado de vermífugos e principalmente a relutância dos produtores em procurar orientação técnica podem favorecer o desenvolvimento de parasitas resistentes a drogas anti-helmínticos, e este fenômeno tem sido relatado no Brasil e no exterior. Como a resistência do parasita é uma característica hereditária, após cada geração pode haver um aumento de parasitas individuais que seriam capazes de sobreviver ao tratamento medicamentoso (Almeida et al., 2010; Amarilho-Silveira et al., 2015; Gárcia et al., 2016; Lara, 2003; Mallmann Júnior et al., 2018).

Madruga et al. (2016) em um experimento em andamento no sul do país, já constataram uma situação de resistência aos princípios ativos testados até o momento, entre eles; Moxidectina, Cloridrato de Levamisol e Albendazole. O resultado pode ser considerado como consequência dos constantes erros de manejo nas propriedades rurais e ao uso indiscriminado de diversos princípios ativos disponíveis nos mercados. O respectivo trabalho até o momento não apresentou resultados de eficiência acima de $95 \%$ em nenhum dos vermífugos utilizados, sendo que de acordo com as recomendações da World Association for the Advancement of Veterinary Parasitology (WAAVP) (Coles et al.,1992) os vermífugos podem ser classificados em: altamente eficaz (mais de 98\%), eficaz (90-98\%), moderadamente eficaz (80-89\%) ou insuficientemente ativo (menos de 80\%). No sul do Espirito Santo foi diagnosticada resistência parasitaria ao princípio ativo ivermectina, nas três propriedades avaliadas, e em duas delas também apresentaram populações de nematoides resistentes a albendazol (Starling et al., 2017). Também foram observados baixos percentuais de eficácia e de redução de OPG em um estudo com o uso da moxidectina, quando comparada à associação albendazole, levamisole e ivermectina em ovinos, sendo 82\% o valor máximo de eficácia alcançado (Buzzulini et al., 2007). Segundo Batista et al. (2017) em duas das quatro propriedades avaliadas em seu estudo, a ivermectina apresentou eficácia abaixo de $69 \%$. Em uma das quatro propriedades também foi testado o albendazol, o qual também apresentou baixo rendimento com eficácia de $60 \%$. Em um estudo conduzido no estado da Bahia, identificou populações dos gêneros Haemonchus e Trichostrongylus resistentes a albendazole, ivermectina, levamisole, moxidectina e closantel, o que sugere resistência anti-helmintica múltipla na média dos rebanhos avaliados no bioma da caatinga e mata atlântica no estado (Borges et al., 2015).

Mallmann Júnior et al. (2018) conduziram um estudo em sete rebanhos no estado do Rio Grande do Sul em regiões distintas, e concluíram que em todos os rebanhos foi diagnosticada resistência ao levamisol, febendazol, moxidectina e closantel, e, em quatro das sete propriedades foi encontrada resistência ao monepantel. Com esse trabalho observaram que os rebanhos gaúchos se encontram em uma situação crítica de resistência anti-helmíntica e que há necessidade de se adotarem outras medidas de controle integrado além do tratamento exclusivo com anti-helmíntico. A resistência parasitária é de grande importância no atual cenário da ovinocultura no Brasil, e o problema vem se agravando pela pressão de seleção dos helmintos resistentes aos fármacos encontrados no mercado, algumas 
estratégias para contornar essa situação estão sendo estudadas entre elas a fitoterapia, a qual pode vir a ser uma boa alternativa no controle desses endoparasitas, além de apresentar baixo custo e uma forma sustentável de eliminar essas cepas resistentes (Amarante, 2009; Chagas \& Veríssimo, 2008; Chagas et al., 2008).

\section{Azadirachta indica, A. Juss (Neem)}

As plantas medicinais durante anos compuseram a base da medicina tradicional, embasada nos conhecimentos culturais de povos de diferentes etnias, passadas entre gerações (Fenalti et al., 2016). A utilização de plantas medicinais tem base no conhecimento familiar e é uma prática na medicina popular, sendo a comunicação oral o mecanismo de transmissão desse conhecimento através do contato dos homens mais velhos com os mais novos (Brasileiro et al., 2008). As substâncias bioativas com potencial fitoterápico são obtidas das plantas ou de seus derivados vegetais, as quais são utilizadas com finalidade profilática, curativa e paliativa (Fenalti et al., 2016). A fitoterapia no controle de verminose é uma alternativa que poderá reduzir o uso de anti-helmínticos sintéticos. Fenalti et al. (2016) descreveram que na medicina veterinária os estudos que abordam a eficácia das plantas medicinais e seu potencial antihelmíntico em pequenos ruminantes vêm ganhando espaço rapidamente em relação aos estudos em humanos, devido ao alto índice de resistência parasitaria para anti-helmínticos comerciais.

Muitas plantas são tradicionalmente conhecidas com potencial de atividade anti-helmíntica, mesmo assim necessita-se de pesquisas para comprovar cientificamente sua eficácia (Vieira, 2008). Entre as várias plantas com esse tipo de potencial destaca-se o neem (Azadirachta indica, A. Juss), planta que vem sendo estudada a partir de seus componentes biológicos, e por possuir vários efeitos benéficos na fitoterapia (Nova et al., 2014; Sczesny-Moraes et al., 2010).

O neem (Figura 3) é uma planta que pertence à família Meliaceae, como o mogno da Amazônia, o cedro, e a Melia Azedarach. Pertecente a ordem dos Rutales, Subordem Rutinaea, Subfamília Melioideae, Tribo Meliae, Gênero Azadirachta e Espécie Azadirachta indica. A Azadirachta indica possui vários nomes comuns pelo mundo, por exemplo, na Índia pode ser chamada de neem, nim, limba, na Austrália e nos Estados Unidos; Neem, na África nim, babo, yaro e marrango, na américa Latina nim, na Espanha e Portugal nim e margosa, na Inglaterra nim e niembaum e no Brasil nim e nime (Brasil, 2013).

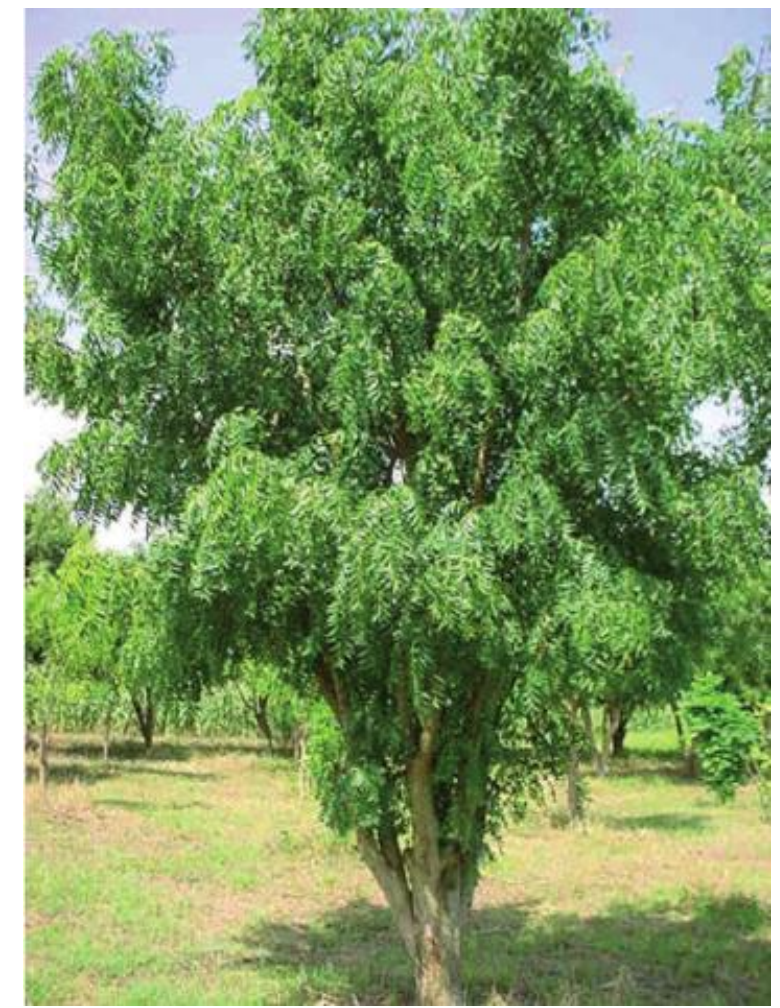

Figura 3. Azadirachta indica, A. Juss (Neem). Fonte: Montes-Molina et al. (2008). 
O neem vem sendo usado no oriente médio a séculos como planta medicinal no tratamento de infecções virais, inflamações, febre e hipertensão. É uma árvore repelente, também sendo usada sua madeira na construção, como combustível, lubrificante, e recentemente como praguicida (Mossini \& Kemmelmeier, 2005). Brasil (2013) cita que o neem é usado como matéria prima nas indústrias de remédios, cosméticos e inseticidas. A árvore desenvolve melhor em regiões com clima tropical e subtropical. Possui um porte de aproximadamente 15 a $20 \mathrm{~m}$ de altura, com tronco semi-ereto a ereto de 20 a $80 \mathrm{~cm}$ de diâmetro, possui fissuras e escamas e tem uma coloração marrom-avermelhada. A copa possui um diâmetro entre 8 a 12 metros podendo chegar a 15 metros em árvores isoladas (Mossini \& Kemmelmeier, 2005). A árvore do neem normalmente começa a fornecer frutos de 3-5 anos após o plantio, com produção superando $25 \mathrm{~kg} /$ planta a partir do quinto ano (Mossini \& Kemmelmeier, 2005). A época de seu florescimento é nos meses de fevereiro a maio e o amadurecimento dos frutos ocorre entre os meses de junho a agosto em área de ocorrência natural (Brasil, 2013), podendo ocorrer uma segunda florada entre novembro a janeiro. A forma de propagação do neem pode ser tanto sexualmente quanto vegetativamente, podendo ser plantado por sementes, mudas, árvores novas, brotos de raiz ou tecido da cultura. Para um bom desenvolvimento, a espécie mostra um bom desempenho em solos arenosos, profundos e bem drenados, com pH de 6,5 a 7,5 e em áreas com chuvas anuais entre 800 a $1800 \mathrm{~mm}$ (Mossini \& Kemmelmeier, 2005). O neem vem se tornando um centro de interesse da comunidade de desenvolvimento científico. A planta vem despertando interesse em cientistas de várias áreas, à medida que seus biocompostos encontram uso eficiente na agricultura, pecuária e medicina. Os compostos extraídos do neem se mostram seletivos, com baixa toxicidade para organismos não alvos, não apresentam características mutagênicas e são rapidamente biodegradados, com mínimo de interferência ao ecossistema (Brasil, 2013; Mossini \& Kemmelmeier, 2005; Schmutterer, 1990). A planta possui mais de 135 compostos distintos divididos em duas classes distintas, sendo a principal classe a dos isoprenóides. Os isoprenóides incluem diterpenóides e triterpenóides, como nimbin, salanin e azadiractina. A azadiractina é o principal limonóide isolado do neem e tem demonstrado várias atividades biológicas altamente eficientes no controle de pragas (Brasil, 2013). A azadiractina causa diversos efeitos nos insetos, como ação detergente, regulação na alimentação, inibe o crescimento, interfere na fecundidade, é anti-opositora e atua na redução da aptidão física do inseto (Brasil, 2013). Especificamente em parasitos a ação dessa substância causa diminuição da ingestão de nutrientes, diminuição da postura de ovos em ectoparasitos, regulação do crescimento, inibição da reprodução, impedimento de movimentação e inibição da síntese de quitina. Essa ação acontece na maioria dos casos nas fases larvais, mas algumas espécies respondem nas suas fases adultas (Mossini \& Kemmelmeier, 2005). A planta também vem sendo muito utilizada na pecuária, pois além da ação repelente contra carrapato e mosca-do-chifre, o neem vem sendo utilizado no controle de sarna, pulgas, piolhos, caramujos, crustáceos, bactérias, vírus, fungos, na cicatrização e assepsia de ferimentos, e como vermífugo (Mossini \& Kemmelmeier, 2005).

Diversos ensaios experimentais são relatados quanto ao emprego de formulações contendo neem no controle de ectoparasitas e endoparasitas em grandes ruminantes. Lipinski et al. (2011) avaliaram a eficácia do neem associado a alho desidratado em bubalino utilizando dois gramas de cada fitoterápico e obtiveram eficiência no controle da ovoposição de nematoides gastrintestinais. Amin et al. (2009) também observaram a eficácia de diversas plantas indianas no controle de nematoides gastrintestinais de bovinos In vitro, dentre as quais, o neem chegou a apresentar eficácia de $100 \%$ em vermes adultos.

Existem poucos trabalhos com utilização dos diferentes subprodutos de neem no controle de endoparasitas em pequenos ruminantes. (Nova et al., 2014; Sczesny-Moraes et al., 2010) não obtiveram resultados satisfatórios com a utilização de óleo de neem via oral, com duas administrações na dosagem de $2,5 \mathrm{ml}$ por quilo de peso vivo, fornecidos em intervalos de 14 dias. Igarashi et al. (2013) constataram que a administração oral de óleo e folhas de neem em ovinos fêmeas nas concentrações $0,5 \mathrm{ml} ; 0,8 \mathrm{ml} ; 1$ $\mathrm{ml} / \mathrm{kg}$ e 1,5g; 2g; 2,5 g/animal respectivamente não foi eficaz no tratamento de nematoides gastrintestinais de ovinos. No entanto, estudos realizados na Embrapa Caprinos com folhas secas do neem, apresentaram como resultado positivo uma ação ovicida em cultura de fezes (Chagas et al., 2008; Fernandes et al., 2010; Srivastava et al., 2008). O Neem (Azadirachta indica) vem se tornando uma importante ferramenta para a área medicinal, agrícola e pecuária, sendo utilizada há séculos pelo povo indiano e nos últimos anos pelo resto do mundo. A planta ainda é digna de estudos para diferentes fins devidos sua variedade de metabólitos secundários (Brasil, 2013). 


\section{Considerações finais}

Considerando a importância da verminose gastrintestinal na produção de caprinos e ovinos, bem como os problemas acima apontados, torna-se necessário investir em pesquisas que visem à busca de alternativas de controle, que sejam de baixo custo e menos nocivas à saúde humana e ao meio ambiente. Dentre essas alternativas, devem-se considerar fitoterápicos com ação anti-helmíntica, entre eles podemos citar a torta de neem.

Espera-se que a utilização da torta de neem seja mais estudada, pois para pesquisa ficar aprovada um resultado satisfatório no controle de endoparasitas em ovinos, tornara-se um método alternativo no tratamento de tais afecções, diminuindo assim, os impactos ambientais e resíduos nos alimentos gerados pelo uso indiscriminado de vermífugos sintéticos.

Resultados sobre a eficiência no controle destes nematoides gastrintestinais com uso da torta de nem, seria um passo para uma produção de animais de forma sustentável, gerando saúde, bem-estar e equilíbrio para o ambiente e o consumidor.

\section{Referências bibliográficas}

Afonso, V. A. C., Costa, R. L. D., Soares Filho, C. V., Cunha, E. A. d., Perri, S. H. V. \& Bonello, F. L. (2013). Supplementation with protected fat to manage gastro-intestinal nematode infection in Santa Ines sheep. Semina. Ciências Agrárias, 34(3):1227-1238.

Almeida, F. A., García, K. C. O. D., Torgerson, P. R. \& Amarante, A. F. T. (2010). Resistência múltipla aos anti-helmínticos por Haemonchus contortus e Trichos trongylus colubriformis em ovinos no Brasil. Parasitologia Internacional, 59(4):622-625.

Amarante, A. F. T. (2009). Nematoides gastrintestinais em ovinos. Doenças parasitárias de caprinos e ovinos: epidemiologia e controle. Brasília: Embrapa Informação Tecnológica, Brasília.

Amarante, A. F. T., Bricarello, P. A., Rocha, R. A. \& Gennari, S. M. (2004). Resistance of Santa Ines, Suffolk and Ile de France sheep to naturally acquired gastrointestinal nematode infections. Veterinary Parasitology, 120(1):91-106.

Amarilho-Silveira, F., Brondani, W. C., Motta, J. F., Ferreira, O. G. L. \& Lemes, J. S. (2015). Resistência ovina frente a nematoides gastrintestinais. Archivos de Zootecnia, 64(247):1-12.

Amin, M. R., Mostofa, M., Hoque, M. E. \& Sayed, M. A. (2009). In vitro anthelmintic efficacy of some indigenous medicinal plants against gastrointestinal nematodes of cattle. Journal of the Bangladesh Agricultural University, 7(1):57-61.

ANUALPEC. (2018). Anuário da Pecuária Brasileira (20th ed. Vol. 1). São Paulo, São Paulo, Brasil: Instituto FNP.

Aquino, R. S., Lemos, C. G., Alencar, C. A., Silva, E. G., Silva Lima, R., Gomes, J. A. F. \& Silva, A. F. (2016). A realidade da caprinocultura e ovinocultura no semiárido brasileiro: um retrato do sertão do Araripe, Pernambuco. PUBVET, 10(4):271-281.

Barbieri, A. M. E., Louvandini, H., Araujo, R. C., Costa, R. L. D., Fornazari, B. C. \& Katiki, L. M. (2014). Potencial anti-helmíntico de formulações contendo óleos essenciais em nematóides gastrintestinais de ovinos. Boletim de Indústria Animal, 7157-57.

Batista, L. F., Ramos, L. F., Brito, S. N. S., Oliveira Castro, A. L., Antunes, C. R. \& Oliveira, L. L. S. (2017). Resistência anti-helmíntica em nematoides gastrintestinais de ovinos. PUBVET, 11(12):1188-1297.

Borges, S. L., Oliveira, A. A., Mendonça, L. R., Lambert, S. M., Viana, J. M., Nishi, S. M., Almeida, M. A. O. (2015). Anthelmintic resistance in goat herds in the Caatinga and Mata Atlântica biomes. Pesquisa Veterinária Brasileira, 35(7):643-648.

Brasil, R. B. (2013). Aspectos botânicos, usos tradicionais e potencialidades de Azadirachta indica (Neem). Enciclopédia Biosfera, 9(17):3252-3258.

Brasileiro, B. G., Pizziolo, V. R., Matos, D. S., Germano, A. M. \& Jamal, C. M. (2008). Plantas medicinais utilizadas pela população atendida no" Programa de Saúde da Família", Governador Valadares, MG, Brasil. Revista Brasileira de Ciências Farmacêuticas, 44(4):629-636. 
Brechelt, A., Taveras, F., Santos, J. A., Pujols, F. M., Peralta Girón, R., Nina, N. \& Montero, A. (1995). El nim: un árbol para la agricultura y el medio ambiente; experiencias en la República Dominicana. Santo Domingo, FRepública Dominicana: Fundación Agricultura y Medio Ambiente.

Buzzulini, C., Silva Sobrinho, A. G., Costa, A. J., Santos, T. R., Borges, F. A. \& Soares, V. E. (2007). Eficácia anti-helmíntica comparativa da associação albendazole, levamisole e ivermectina a moxidectina em ovinos. Pesquisa Agropecuária Brasileira, 42(6):891-895.

Chagas, A. C. S. (2004). Controle de parasitas utilizanto extratos vegetais. Revista Brasileira de Parasitologia Veterináia, 13(Sup. 1):156-160.

Chagas, A. C. S. \& Veríssimo, C. J. (2008). Principai s enfermidades e manejo sanitário de ovinos. Embrapa Pecuária Sudeste, 1(1):70.

Chagas, A. C. S., Vieira, L. S., Freitas, A. R., Araújo, M. R. A., Araújo-Filho, J. A., Araguão, W. R. \& Navarro, A. M. C. (2008). Anthelmintic efficacy of neem (Azadirachta indica A. Juss) and the homeopathic product Fator Vermes ${ }^{\circledR}$ in Morada Nova sheep. Veterinary Parasitology, 151(1):68-73.

Coles, G.C., Bauer, C., Borgsteede, F.H.M., Klei, T.R., Taylor, M.A., Waller, P.J., (1992). World Association for the Advancement of Veterinary Parasitology (W.A.A.V.P.) methods for the detection of anthelmintic resistance in nematodes of veterinary importance. Vet. Parasitol.44, 35-44.

Dias, A. S., Melotti, V. D., Serrano, D. H., Altoé, G., Spadetto, R. M., Aguiar, G. B., Sobreira, R. R. (2014). Avaliação da eficácia de uma formulação comercial contendo torta de nim no controle de nematoides gastrintestinais de equinos. Acta Veterinaria Brasilica, 8(3):186-191.

Fenalti, J. M., Santos, T. M., Santos, P. C., Baccega, B. B. \& Scaini, C. J. (2016). Diversidade das plantas brasileiras com potencial anti-helmíntico. Vittalle-Revista de Ciências da Saúde, 28(1):3849.

Fernandes, J. I., Correia, T. R., Ribeiro, F. A., Cid, Y. P., Tavares, P. V. \& Scott, F. B. (2010). Eficácia in vitro do nim (Azadirachta indica) no controle de Rhipicephalus sanguineus (Latreille, 1806)(Acari: Ixodidae). Revista Brasileira de Medicina Veterinária, 32(Supl 1):64-68.

Fortes, F. S. \& Molento, M. B. (2013). Resistência anti-helmíntica em nematoides gastrintestinais de pequenos ruminantes: avanços e limitações para seu diagnóstico. Pesquisa Veterinária Brasileira, 33(12):1391-1402.

Gárcia, C., Sprenger, L. K., Ortiz, E. B. \& Molento, M. B. (2016). First report of multiple anthelmintic resistance in nematodes of sheep in Colombia. Anais da Academia Brasileira de Ciências, 88(1):397402.

Igarashi, M., Carvalho, D. M. G., Bucci, F. C., Miranda, Y., Rodrigues, Z. M., Almeida, M. C. F. \& Piona, M. N. M. (2013). Efeito do Neem (Azadirachta indica) no controle de nematódeos gastrintestinais em ovinos suplementados a pasto no período seco. Semina: Ciências Agrárias, 34(1):301=310.

Jimenez-Sanz, A. L., Quirino, C. R., Pacheco, A., Costa, R. L. D., Beltrame, R. T., Rua, M. A. S., Madella-Oliveira, A. F. (2016). Relação entre fatores associados às parasitoses gastrointestinais, desempenho e estado fisiológico de ovelhas Santa Inês. Agropecuária Técnica, 37(1):88-95.

Kudke, R., Kalaskar, S. \& Nimbalkar, R. (1999). Neem leaves as feed supplement for livestock. Pushudhn, 1412-14.

Lara, D. M. (2003). Resistencia a los antihelmínticos: origen, desarrollo y control. Ciencia y Tecnología Agropecuaria, 4(1):55-71.

Levine, N. D. (1973). Protozoan parasites of domestics animals and man (Vol. 2). Mineapolis: Borges Publishing Company.

Lipinski, L. C., Martinez, J. L., Santos, M. V. R., Ferreira, J. N. \& Pfau, D. R. (2011). Avaliação do efeito anti-helmíntico e das alterações metabólicas em búfalos (Bubalus bubalis) com administração da torta de neem e do alho desidratado no sul do Paraná. Revista Brasileira de Agroecologia, 6(3):168-175.

Madruga, A. M., Corrêa, G. F., Chagas, R. A., Bianchini, B. D., Santos, P. M. \& Lopes, P. R. S. (2016). Avaliação da eficácia de anti-helmínticos e da resistência parasitária em ovinos. Anais do Salão Internacional de Ensino, Pesquisa e Extensão, 7(2):1-2. 
Mallmann Júnior, P. M., Raimondo, R. F., Rivero, B. R. C., Jacondino, L. R., Gonçalves, A. S., Silveira, B. O. \& Oberst, E. R. (2018). Resistance to monepantel in multiresistant gastrointestinal nematodes in sheep flocks in Rio Grande do Sul. Semina: Siências Agrárias, 39(5):2019-2070.

Montes-Molina, J. A., Luna-Guido, M., Ceballos-Ramirez, J. M., Fernández-Luqueño, F., EspinozaPaz, N., Rincón-Rosales, R., Gutierrez-Miceli, F. A. (2008). Effect of pest-controlling neem and mata-raton on bean growth, soil N and soil CO 2 emissions. Agronomy for Sustainable Development, 28(2):187-194.

Mossini, S. A. G. \& Kemmelmeier, C. A. (2005). A árvore Nim (Azadirachta indica A. Juss): múltiplos usos. Acta Farmaceutica Bonaerense, 24(1):139-148.

Nova, L. E. V., Costa, M. E., Melo, P. G. C. F., Cunha Filho, L. F. C., Junior, F. A. B., Silva, L. C., Bogado, A. L. G. (2014). Resistência de nematoides aos anti-helmínticos nitroxinil $34 \%$ e ivermectina $1 \%$ em rebanho ovino no município de São João do Ivaí, Paraná. Revista Brasileira de Higiene e Sanidade Animal, 8(1):159-171.

Roeber, D. L., Cannell, R. C., Belk, K. E., Miller, R. K., Tatum, J. D. \& Smith, G. C. (2000). Implant strategies during feeding: impact on carcass grades and consumer acceptability. Journal of Animal Science, 78(7):1867-1874.

Roeber, F., Jex, A. R. \& Gasser, R. B. (2013). Impact of gastrointestinal parasitic nematodes of sheep, and the role of advanced molecular tools for exploring epidemiology and drug resistance-an Australian perspective. Parasites \& Vectors, 6(153):1-18.

Schmutterer, H. (1990). Properties and potential of natural pesticides from the neem tree, Azadirachta indica. Annual Review of Entomology, 35(1):271-297.

Sczesny-Moraes, E. A., Bianchin, I., Silva, K. F. d., Catto, J. B., Honer, M. R. \& Paiva, F. (2010). Resistência anti-helmíntica de nematóides gastrintestinais em ovinos, Mato Grosso do Sul. Pesquisa Veterinária Brasileira, 30(3):239-236.

Sorio, A. \& Rasi, L. (2010). Ovinocultura e abate clandestino: um problema fiscal ou uma solução de mercado? Revista de Política Agrícola, 19(1):71-83.

Sotomaior, C. S., Carli, L. M., Tangleica, L., Kaiber, B. K. \& Souza, F. P. (2007). Identificação de ovinos e caprinos resistentes e susceptíveis aos helmintos gastrintestinais. Revista Acadêmica, 5(4):397-412.

Srivastava, R., Ghosh, S., Mandal, D. B., Azhahianambi, P., Singhal, P. S., Pandey, N. N. \& Swarup, D. (2008). Efficacy of Azadirachta indica extracts against Boophilus microplus. Parasitology Research, 104149-153.

Starling, R. Z. C., Alves, C. S., Viana, M. V. G. \& Dietrich, W. S. (2017). Diagnóstico in vivo da sensibilidade de nematoides a diferentes antihelmínticos em ovinos criados em sistema semiintensivo. Archives of Veterinary Science, 22(2):38-47.

Urquhart, G. M. (1996). Parasitologia veterinária (2 ed.). Rio de Janeiro: Guanabara Koogan.

Viana, J. G. A. (2008). Panorama geral da ovinocultura no mundo e no Brasil. Revista Ovinos, 4(12):19.

Viana, J. G. A., Moraes, M. R. E. \& Dorneles, J. P. (2015). Dinâmica das importações de carne ovina no Brasil: análise dos componentes temporais. Semina: Ciências Agrárias, 36(3):2223-2234. doi: http:dx.doi.org/10.5433/1679-0359.2015v36n3Supl1p2223.

Vieira, L. S. (2008). Métodos alternativos de controle de nematóides gastrintestinais em caprinos e ovinos. Revista de Tecnologia e Ciências Agropecuária, 2(2):49-56.

Recebido: 10 de março, 2019.

Aprovado: 1 de abril, 2019.

Publicado: 15 de abril, 2019.

Licenciamento: Este artigo é publicado na modalidade Acesso Aberto sob a licença Creative Commons Atribuição 4.0 (CC-BY 4.0), a qual permite uso irrestrito, distribuição, reprodução em qualquer meio, desde que o autor e a fonte sejam devidamente creditados. 